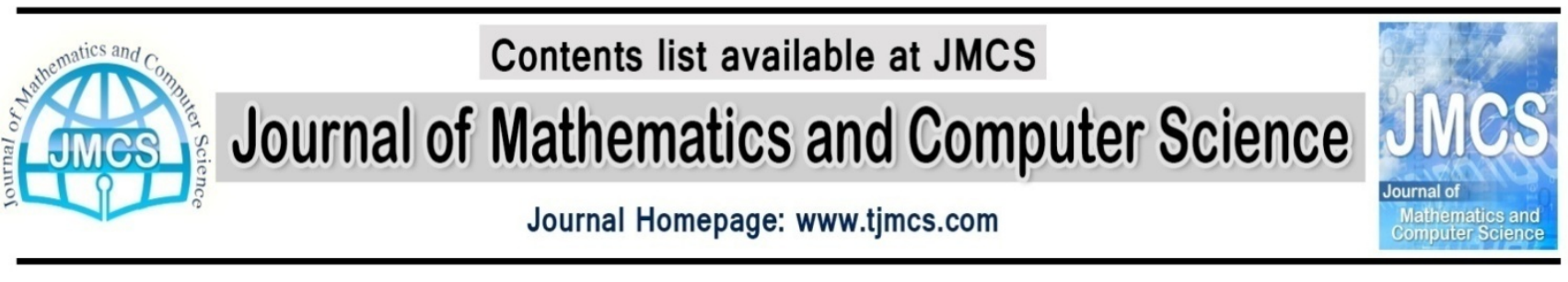

\title{
A New Modified Approach for solving seven-order Sawada-Kotara equations
}

Article history:

\author{
Masoud Saravi ${ }^{1}$, Ali Nikkar ${ }^{2 *}$, Martin Hermann ${ }^{3}$, Javad Vahidi ${ }^{4}$, Reza Ahari ${ }^{5}$ \\ ${ }^{1}$ Department of mathematics, Islamic Azad University, Nour Branch, Nour, Iran \\ ${ }^{2}$ Department of Civil Engineering, University of Tabriz, Tabriz, Iran \\ ${ }^{3}$ Fakultätfür Mathematik und Informatic, Friedrich-Schiller-Universität Jena, Germany \\ ${ }^{4}$ Department of mathematics, Iran University of Science and Technology, Tehran, Iran \\ ${ }^{5}$ School of Electrical and Computer Engineering, University of Shiraz, Shiraz, Iran
}

Received January 2013

Accepted February 2013

Available online April 2013

\section{msaravi2001@yahoo.com,ali.nikkar@yahoo.com}

\begin{abstract}
Herein, Reconstruction of Variational Iteration Method (RVIM) is used for computing solutions of the seventh-order Sawada-Kotera equation (sSK) and a Lax's seventh order KdV equations (LsKdV). The results are compared with the Adomian decomposition method (ADM) and the known analytical solutions. Results obtained expose effectiveness and capability of this method to solve the seven-order Sawada-Kotera (sSK) and a Lax's seven-order KdV (LsKdV) equations.
\end{abstract}

Keywords: Reconstruction of Variational Iteration Method (RVIM), seven-order Sawada-Kotera (sSK), Lax's seven-order KdV (LsKdV), Adomian decomposition method (ADM).

\section{Introduction}

Establishing a new reliable algorithm of variational iteration method, Reconstruction of variational iteration method is the purpose of this paper. In various areas of science, especially in physics and engineering, variational methods were considered [1, 2]. The variational iteration method was proposed by $\mathrm{He}$ [3-5] and was successfully applied to autonomous ordinary differential equation [6], to Burger's and coupled Burger's equations [7], to Bratu's equation [8], to non-linear partial differential equations with variable coefficients [9], to linear Helmholtz partial differential equation [10], and to Klein-Gordon equations [11]. It was shown that this method is more powerful than existing techniques such as the Adomian method [12, 13], homotopy perturbation method [14] Energy Balance method [15], Homotopy Analysis Method [16], etc. The perturbation method suffers from the computational workload, especially when the degree of nonlinearity increases. Moreover, the Adomian method suffers from the complicated algorithms used to calculate the Adomian polynomials that are necessary for nonlinear problems. 
In 2009 Hesameddini and Latifizadeh proposed a new method based on Laplace transform Reconstruction of variational iteration method (RVIM) [17, 18, 24-26] in which the correctional function of the variational iteration method is obtained without using the Variational Theory [3-5]. Therefore in this method the complexity in calculating the Lagrange multiplier has been removed. The RVIM technique has no specific requirements, such as linearization, small parameters, etc.

In this paper, RVIM is used for studying the following seventh order Sawada-Kotera equation and Lax's seventh-order KdV equation.

$$
\begin{gathered}
u_{t}+\left(35 u^{4}+70\left(u^{2} u_{x x}+u u_{x}^{2}\right)+7\left(2 u u_{x x x x}+3 u_{x x}^{2}+4 u_{x} u_{x x x}\right)+u_{x x x x x x}\right)_{x}=0 \\
u_{t}+\left(63 u^{4}+63\left(2 u^{2} u_{x x}+u u_{x}^{2}\right)+21\left(u u_{x x x x}+u_{x x}^{2}+u_{x} u_{x x x}\right)+u_{x x x x x x}\right)_{x}=0 .
\end{gathered}
$$

Eq. (1) is known as the seventh order Sawada -Kotera equation $[19,20]$ and Eq. (2) is known as the Lax's seventh-order KdV equation $[19,21]$ respectively. Further we compare the result with given solutions using ADM $[19,22]$.

\section{Basic Idea of RVIM}

To clarify the basic ideas of our proposed method in [17], we consider the following differential equation same as VIM based on Lagrange multiplier [23]:

$$
\operatorname{Lu}\left(x_{1}, \cdots, x_{k}\right)+N u\left(x_{1}, \cdots, x_{k}\right)=f\left(x_{1}, \cdots, x_{k}\right)
$$

By suppose that

$$
L u\left(x_{1}, \cdots, x_{k}\right)=\sum_{i=0}^{k} L_{x i} u\left(x_{i}\right)
$$

Where $L$ is a linear operator, $N$ a nonlinear operator and $f\left(x_{1}, \cdots, x_{k}\right)$ an inhomogeneous term.

We can rewrite equation (3) down a correction functional as follows:

$$
L_{x j} u\left(x_{j}\right)=\underbrace{f\left(x_{1}, \cdots, x_{k}\right)-N u\left(x_{1}, \cdots, x_{k}\right)-\sum_{i \neq j}^{k} L_{x i} u\left(x_{i}\right)}_{h\left(\left(x_{1}, \cdots, x_{k}\right), u\left(x_{1}, \cdots, x_{k}\right)\right)}
$$

therefore

$$
L_{x j} u\left(x_{j}\right)=h\left(\left(x_{1}, \cdots, x_{k}\right), u\left(x_{1}, \cdots, x_{k}\right)\right)
$$

With artificial initial conditions being zero regarding the independent variable $x_{j}$.

By taking Laplace transform of both sides of the equation (6) in the usual way and using the artificial initial conditions, we obtain the result as follows

$$
P(s) . U\left(x_{1}, \cdots, x_{i-1}, s, x_{i+1}, x_{k}\right)=H\left(\left(x_{1}, \cdots, x_{i-1}, s, x_{i+1}, x_{k}\right), u\right)
$$

Where $\mathrm{P}(\mathrm{s})$ is a polynomial with the degree of the highest derivative in equation (7), (the same as the highest order of the linear operator $L_{x_{j}}$ ). The following relations are possible;

$$
\ell[\mathrm{h}]=\mathrm{H}
$$




$$
\begin{aligned}
& \mathrm{B}(\mathrm{s})=\frac{1}{\mathrm{P}(\mathrm{s})} \\
& \ell\left[\mathrm{b}\left(\mathrm{x}_{\mathrm{i}}\right)\right]=\mathrm{B}(\mathrm{s})
\end{aligned}
$$

Which that in equation (8-a) the function $H\left(\left(x_{1}, \cdots, x_{i-1}, s, x_{i+1}, x_{k}\right), u\right)$ an $h\left(\left(x_{1}, \cdots, x_{i-1}, x_{i}, x_{i+1}, x_{k}\right), u\right)$ have been abbreviated as $H, h$ respectively.

Hence, rewrite the equation (7) as;

$$
U\left(x_{1}, \cdots, x_{i-1}, s, x_{i+1}, x_{k}\right)=H\left(\left(x_{1}, \cdots, x_{i-1}, s, x_{i+1}, x_{k}\right), u\right) \cdot B(s)
$$

Now, by applying the inverse Laplace Transform on both sides of equation (9) and by using the (8-a) - (8c), we have;

$$
\mathrm{u}\left(\mathrm{x}_{1}, \cdots, \mathrm{x}_{\mathrm{i}-1}, \mathrm{x}_{\mathrm{i}}, \mathrm{x}_{\mathrm{i}+1}, \mathrm{x}_{\mathrm{k}}\right)=\int_{0}^{\mathrm{x}_{\mathrm{i}}} \mathrm{h}\left(\left(x_{1}, \cdots, x_{i-1}, \tau, x_{i+1}, x_{k}\right), u\right) \cdot b\left(x_{i}-\tau\right) d \tau
$$

Now, we must impose the actual initial conditions to obtain the solution of the equation (3). Thus, we have the following iteration formulation:

$$
\begin{aligned}
\mathrm{u}_{\mathrm{n}+1}\left(\mathrm{x}_{1}, \cdots, \mathrm{x}_{\mathrm{i}-1}, \mathrm{x}_{\mathrm{i}}, \mathrm{x}_{\mathrm{i}+1}, \mathrm{x}_{\mathrm{k}}\right)= & \mathrm{u}_{0}\left(\mathrm{x}_{1}, \cdots, \mathrm{x}_{\mathrm{i}-1}, \mathrm{x}_{\mathrm{i}}, \mathrm{x}_{\mathrm{i}+1}, \mathrm{x}_{\mathrm{k}}\right) \\
& +\int_{0}^{\mathrm{x}_{\mathrm{i}}} \mathrm{h}\left(\left(x_{1}, \cdots, x_{i-1}, \tau, x_{i+1}, x_{k}\right), u\right) \cdot b\left(x_{i}-\tau\right) d \tau
\end{aligned}
$$

where $\mathrm{u}_{0}$ is initial solution with or without unknown parameters. Assuming $\mathrm{u}_{0}$ is the solution of, $L u$, with initial/boundary conditions of the main problem, In case of no unknown parameters, $u_{0}$ should satisfy initial/boundary conditions. When some unknown parameters are involved in $\mathrm{u}_{0}$, the unknown parameters can be identified by initial/boundary conditions after few iterations, this technology is very effective in dealing with boundary problems. It is worth mentioning that, in fact, the Lagrange multiplier in the He's variational iteration method is $\lambda(\tau)=b\left(x_{i}-\tau\right)$ as shown in [17].

The initial values are usually used for selecting the zeroth approximation $\mathrm{u}_{0}$. With $\mathrm{u}_{0}$ determined, then several approximations $\mathrm{u}_{\mathrm{n}} n>0$, follow immediately. Consequently, the exact solution may be obtained by using

$$
\mathrm{u}\left(\mathrm{x}_{1}, \cdots, \mathrm{x}_{\mathrm{i}-1}, \mathrm{x}_{\mathrm{i}}, \mathrm{x}_{\mathrm{i}+1}, \mathrm{x}_{\mathrm{k}}\right)=\lim _{\mathrm{n} \rightarrow \infty} \mathrm{u}_{\mathrm{n}}\left(\mathrm{x}_{1}, \cdots, \mathrm{x}_{\mathrm{i}-1}, \mathrm{x}_{\mathrm{i}}, \mathrm{x}_{\mathrm{i}+1}, \mathrm{x}_{\mathrm{k}}\right) .
$$

\section{Applying RVIM for sSK and LsKdV}

To demonstrate the effectiveness of the method we consider here Eqs.(1) and (2) with given initial condition.

\subsection{Example 1:}

Consider the SSK equation (1) with the initial condition [19]:

$$
u_{0}(x, t)=\frac{4}{3} k^{2}\left(2-3 \tanh ^{2}(k x)\right),
$$

At first rewrite eq. (1) based on selective linear operator as 


$$
\ell\{u(x)\}=u_{t}=\overbrace{-\left(35 u^{4}+70\left(u^{2} u_{x x}+u u_{x}^{2}\right)+7\left(2 u u_{x x x x}+3 u_{x x}^{2}+4 u_{x} u_{x x x}\right)+u_{x x x x x x}\right)_{x}}^{h(x, t, u)}
$$

Now Laplace transform is implemented with respect to independent variable $x$ on both sides of eq. (14) and by using the new artificial initial condition (which all of them are zero) we have

$$
\begin{gathered}
s \cup(x, t)=\ell\{h(x, t, u)\} \\
\cup(x, t)=\frac{\ell\{h(x, t, u)\}}{s}
\end{gathered}
$$

And whereas Laplace inverse transform of $1 / s$ is as follows

$$
\ell^{-1}\left[\frac{1}{S}\right]=1
$$

Therefore by using the Laplace inverse transform and convolution theorem it is concluded that

$$
u(x, t)=\int_{0}^{t} h(x, \varepsilon, u) d \varepsilon
$$

Hence, we arrive the following iterative formula for the approximate solution of subject to the initial condition (13).

So, in exchange with applying recursive algorithm, following relations are achieved

$u_{n+1}=u_{0}+\int_{0}^{t}\left(-\left(35 u_{n}^{4}+70\left(u_{n}^{2} u_{n_{X X}}+u_{n} u_{n_{X}}^{2}\right)+7\left(2 u_{n} u_{n_{X X X X}}+3 u_{n_{X X}}^{2}+4 u_{n_{X}} u_{n_{X X X}}\right)+u_{n_{X X X X X X}}\right)_{x}\right) d \varepsilon$

Now we start with an arbitrary initial approximation $u_{0}(x, t)=\frac{4}{3} k^{2}\left(2-3 \tanh ^{2}(k x)\right)$, that satisfies the initial condition and by using the RVIM iteration formula (19), we have the following successive approximation

$u_{1}(x, t)=\frac{4}{3} k^{2}\left(2-3 \tanh ^{2}(k x)\right)+\frac{1}{9} k^{8} \sec ^{2}(k x) t[2176-896 \cosh (2 k x)]$, 
$u_{2}(x, t)=\frac{4}{3} k^{2}\left(2-3 \tanh ^{2}(k x)\right)+\frac{1}{9} k^{8} \operatorname{sech}^{2}(k x) t[2176-896 \cosh (2 k x)]+$

$\frac{1}{27} \operatorname{sech}^{2}(k x) k^{14} t^{2}([6328576-6566144 \cosh 9(2 k x)+1077248 \cosh (4 k x)+24832 \cosh (6 k x)-$

$12544 \cosh (8 k x)]+\frac{1}{3} k^{6} t[2544812032-2746548224 \cosh (2 k x)+305070080 \cosh (4 k x)+$

$41746432 \cosh (6 k x)-5619712 \cosh (8 k x)]+\frac{1}{9} k^{12} t^{2}[50980192256-23855104000 \cosh (2 k x)-$ $55593402368 \cosh (4 k x)+17983078400 \cosh (6 k x)-1258815488 \cosh (8 k x)]+$ $\left.\frac{1}{135} k^{18} t^{3}[-238459436400640+291359575506944 \cosh (6 k x)-563949338624 \cosh (8 k x)]\right)$
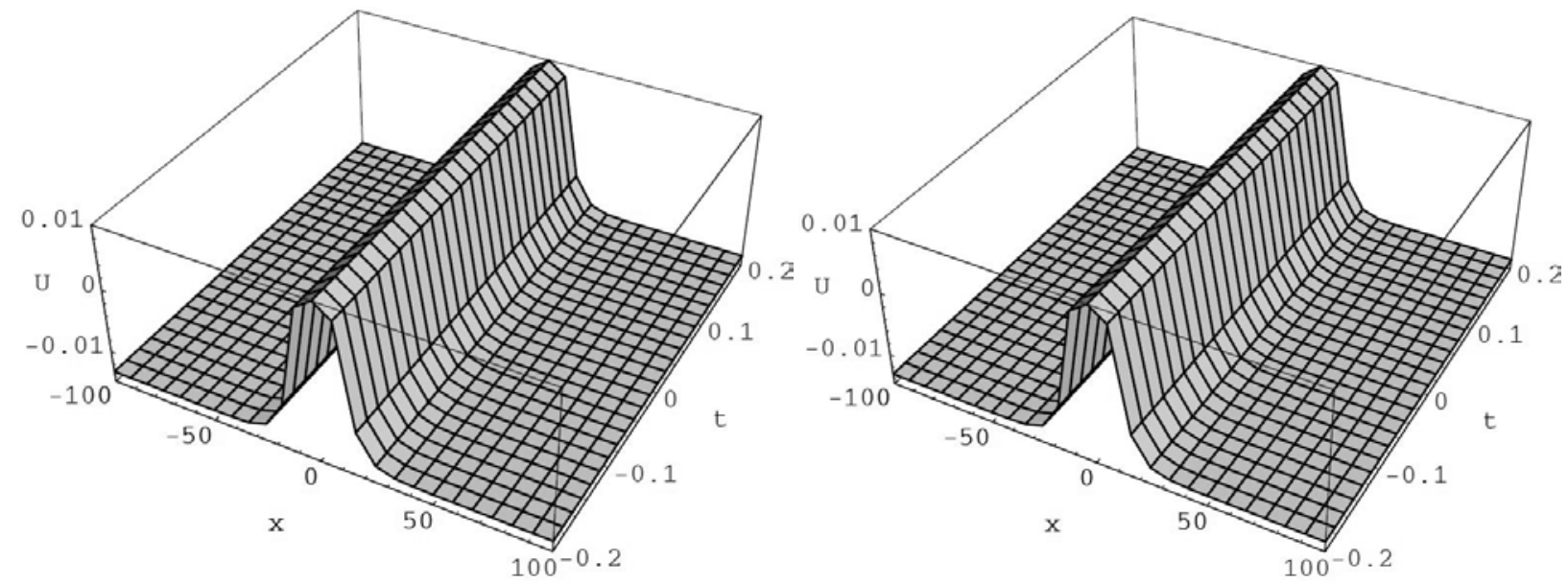

Figure.1. The surfaces on both columns respectively show the solutions, $u(x, t)$, for RVIM on the right and ADM on the left when $k=0.1$.

\subsection{Example 2:}

Consider the LsKdV equation with given initial condition [19]:

$$
u_{0}(x, t)=2 k^{2} \operatorname{sech}^{2}(k x)
$$

At first rewrite eq. (2) based on selective linear operator as

$$
\ell\{u(x)\}=u_{t}=\overbrace{-\left(35 u^{4}+70\left(u^{2} u_{x x}+u u_{x}^{2}\right)+7\left(2 u u_{x x x x}+3 u_{x x}^{2}+4 u_{x} u_{x x x}\right)+u_{x x x x x}\right)_{x}}^{h(x, t, u)}
$$

RVIM's iteration formulae in t-direction can be readily obtained.

$u_{n+1}=u_{0}+\int_{0}^{t}\left(-\left(35 u_{n}^{4}+70\left(u_{n}^{2} u_{n_{X X}}+u_{n} u_{n_{X}}^{2}\right)+7\left(2 u_{n} u_{n_{X X X X}}+3 u_{n_{X X}}^{2}+4 u_{n_{X}} u_{n_{X X X}}\right)+u_{n_{X X X X X}}\right)\right) d \varepsilon$ 
Now we start with an arbitrary initial approximation $u_{0}(x, t)=2 k^{2} \sec h^{2}(k x)$, that satisfies the initial condition and by using the RVIM iteration formula (26), we have the following successive approximation

$$
\begin{aligned}
& u_{1}(x, t)=2 k^{2} \operatorname{sech}^{2}(k x)-128 k^{8} t \sec ^{2}(k x), \\
& u_{2}(x, t)=2 k^{2} \sec ^{2}(k x)-128 k^{8} t \sec ^{2}(k x)-\frac{128}{3} \sec ^{8}(k x) k^{14} t^{2}\left(44040192 t^{3} k^{18}+1720320 t^{2} k^{12}+\right. \\
& 30464 t k^{6}+\left(-3440640 t^{2} k^{12}-7168 t k^{6}+1803\right) \cosh (2 k x)+ \\
& \left.36\left(448 k^{6} t-11\right) \cosh (4 k x)-3 \cosh (6 k x)-2004\right)
\end{aligned}
$$

and so on. Using the above terms, in Fig.2, $u_{2}(x, t)$ and exact solution $u(x, t)=2 k^{2} \operatorname{sech}^{2}(k x)$ [21] is drawn.
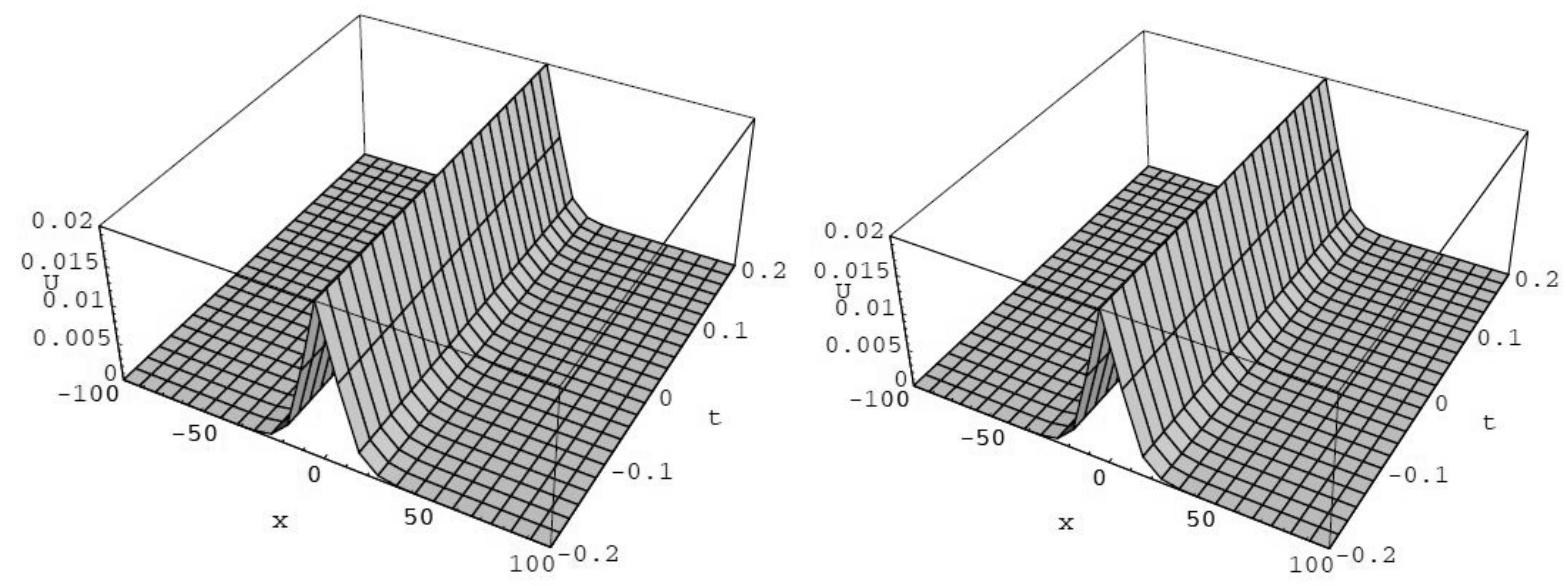

Figure.2. The surfaces on both columns respectively show the solutions, $u(x, t)$, for RVIM on the right and $A D M$ on the left when $\mathrm{k}=0.1$.

\section{Conclusion}

In this paper, we successfully apply Reconstruction of Variational Iteration Method (RVIM) to approximate the solution of SSK and LSKdV equations. Also, comparisons were made between Reconstruction of Variational Iteration Method (RVIM) and Adomian decomposition method (ADM) for SSK and LsKdV equations. Moreover, the RVIM reduces the size of calculations by not requiring the tedious Adomian polynomials, and hence the iteration is direct and straightforward. The results reported here provide further evidence of the usefulness of RVIM for finding the analytic and numeric solutions for the linear and nonlinear diffusion equations and, it is also a promising method to solve different types of nonlinear equations in mathematical physics.

\section{References}

[1] J. Mosler J, M. Ortiz, On the numerical implementation of variational arbitrary Lagrangian-Eulerian 
(VALE) formulations, International Journal for Numerical Methods in Engineering, 67(9), 1272-1289, (2006).

[2] P. Thoutireddy, M. Ortiz, Variational r-adaption and shape-optimization method for finitedeformation elasticity, International Journal for Numerical Methods in Engineering, 61(1), 1-21, (2004).

[3] J. H. He, Approximate analytical solution for seepage flow with fractional derivatives in porous media, Computer Methods in Applied Mechanics and Engineering, 167, 57-68, (1998).

[4] R. Seaadati, M. Dehghan, S.M. Vaezpour, M. Saravi., The convergence of He's variational iteration method for following integral equations, Computers \& Mathematics with Applications, 58, 2167 2171, (2009).

[5] A. Nikkar, M. Mighani, Application of He's variational iteration method for solving seventh-order differential equations, American Journal of Computational and Applied Mathematics, 2(1), 37-40, (2012).

[6] J. H. He, Approximate solution of nonlinear differential equations with convolution product nonlinearities. Computer Methods in Applied Mechanics and Engineering, 167, 69-73, (1998).

[7] M.A. Abdou, A.A. Soliman, Variational iteration method for solving Burgers' and coupled Burgers' equation, J. Comput. Appl. Math., 181, 245-251, (2005).

[8] M. Saravi, M. Hermann, D. Kaiser, Solution of Bratu's Equation by He's Variational Iteration Method, American Journal of Computational and Applied Mathematics, 3(1), 46-48, (2013).

[9] J. H. He Variational principle for some nonlinear partial differential equations with variable coefficients, Chaos, Solitons and Fractals, 19, 847-851, (2004).

[10] S. Momani, S. Abusaad, Application of He's variational-iteration method to Helmholtz equation, Chaos Solitons Fractals, 27(5), 1119-1123, (2005).

[11] S. Abbasbandy, Numerical solution of nonlinear Klein-Gordon equations by variational iteration method, International Journal for Numerical Methods in Engineering, 70, 876-881, (2007).

[12] G. Adomian, Solving Frontier Problems of Physics: The Decomposition Method, Kluwer, Boston, (1994).

[13] G. Adomian, A review of the decomposition method in applied mathematics, Journal of Math. Anal. Appl., 135, 501-544, (1988).

[14] M. Saravi, M. Hermann and H. Ebrahimi Khah, The comparison of homotopy perturbation method with finite difference method for determination of maximum beam deflection, Journal of Theoretical and Applied Physics, 7:8, (2013), doi:10.1186/2251-7235-7-8 
[15] A. Nikkar, S. Esmaeilzade Toloui, K. Rashedi and H. R. Khalaj Hedayati, Application of energy balance method for a conservative X1/3 force nonlinear oscillator and the Doffing equations, International Journal of Numerical Methods and Applications, 5(1), 57-66, (2011).

[16] Y. Khan, R. Taghipour, M. Falahian, A. Nikkar, A new approach to modified regularized long wave equation , Neural Comput \& Applic, (2012), DOI 10.1007/s00521-012-1077-0

[17] E. Hesameddini, H. Latifizadeh, Reconstruction of Variational Iteration Algorithms using Laplace Transform, Internat. J. Nonlinear Sci. Numer. Simulation, 10(10), 1365-1370, (2009).

[18] A. Nikkar, Z. Mighani, S.M. Saghebian, S.B. Nojabaei and M. Daie. Development and Validation of an Analytical Method to the Solution of Modelling the Pollution of a System of Lakes, Research Journal of Applied Sciences, Engineering and Technology, 5(1), 296-302, (2013).

[19] S. M. El-Sayed and D. Kaya, An application of the ADM to seven order Sawada-Kotera equations, Appl. Math. Comput., 157, 93-101, (2004).

[20] W. Hereman, P.P. Banerjee, A. Korpel, G. Assanto, A. van Immerzeele and A. Meerpoel, Exact solitary wave solutions of nonlinear evolution and wave equations using a direct algebraic method, J. Phys. A: Math. Gen., 19, 607-628, (1986).

[21] E. J. Parkes and B.R. Duffy, An automated tanh-function method for finding solitary wave solutions to non-linear evolution equations, Comput. Phys. Commun., 98, 288-300, (1996).

[22] G. Adomian, Solving Frontier Problems of Physics: The Decomposition Method, Kluwer, (1994).

[23] A.M. Wazwaz, The variational iteration method: A powerful scheme for handling Linear and nonlinear diffusion equations, Computers and Mathematics with Applications, 54(7), 933-939, (2007).

[24] A. Nikkar, A new approach for solving Gas dynamics equation. Acta Technica Corviniensis-Bulletin of Engineering, 4, 113-116, (2012).

[25] A. Nikkar, J. Vahidi, M. Jafarnejad Ghomi, M. Mighani, Reconstruction of variation Iteration Method for Solving Fifth Order Caudrey-Dodd-Gibbon (CDG) Equation, International journal of Science and Engineering Investigations, 1(6), 38-41, (2013).

[26] S. Ghasempoor, J. Vahidi, A. Nikkar, M. Mighani, Analytical approach to some highly nonlinear equations by means of the RVIM, Res. J. Appl. Sci. Eng. Technol., 5(1), 296-302, (2013). 